\title{
STUDIES ON SEASONAL VARIATION OF GROUND WATER QUALITY USING MULTIVARIATE ANALYSIS FOR BIDAR URBAN \& ITS INDUSTRIAL AREA (KARNATAKA-STATE, INDIA)
}

\author{
Shivasharanappa ${ }^{1}$, Padaki Srinivas ${ }^{2}$ \\ ${ }^{1}$ Associate Professor, Dept. of Civil Engineering, ${ }^{2}$ Professor \& Head, Chemistry Dept., P. D. A. College of Engineering \\ (Autonomous Institution) Gulbarga, Karnataka State, India. \\ shivasharanappa.g@gmail.com, shiva_patil11@rediffmail.com
}

\begin{abstract}
In this study the seasonal variability of groundwater quality parameters in Bidar urban and its industrial area are investigated. Three water samples each from 35 wards were collected and subjected for physico-chemical analysis. Average of the three samples represents each ward data. Analysis was done for pre-monsoon and post-monsoon seasons of the years 2009, 2010 \& 2011. Seventeen physicochemical parameters viz., pH, total hardness, calcium, magnesium, chloride, nitrate, sulfate, total dissolved solids, iron, fluoride, sodium, potassium, alkalinity, manganese, zinc, dissolved oxygen \& total solids were analyzed. Factor analysis is applied on the data set to investigate the origin of the water pollution sources. FA yielded three factors for each season (combined pre-monsoon \& combined post-monsoon) with $62.8 \%$ and $61.6 \%$ total variance respectively; in addition FA identifies anthropogenic factor (i.e., industrial, urban sewage and agricultural drainage) and natural factor (top soil mixture with water and percolation, weathering of ground strata) as latent pollution sources. Hierarchical cluster analysis grouped 35 sampling stations of Bidar urban into three clusters, i.e., relatively less polluted (LP), and moderately polluted (MP) and highly polluted (HP) sites, based on the similarity of water quality characteristics.
\end{abstract}

Keywords: Bidar urban \& its industrial area, Ground water quality, Correlation coefficients, Factor analysis, Cluster analysis.

\section{INTRODUCTION}

Urbanization and industrial development in the developing countries during the last decade have provoked some serious concern for the environment [1]. Human activities such as intensive agriculture around the out skirts of cities, urbanization and industrialization contribute to ground water deterioration [2]. Ground water quality in cities is a matter of serious concern. Ground water due to the percolation of the domestic and industrial wastewater and run-off from agricultural land are among the most vulnerable water bodies to pollution [3]. A groundwater system carrying the one-way of a significant load of matter both natural and anthropogenic sources [4]. Groundwater is heterogeneous at different spatial scale which may be attributed to a number of factors including anthropogenic input, biomass characteristic, weathering of crustily minerals, local environmental conditions, wastewater discharges, and wastewater percolations [5, 6]. Bidar city and its industrial area is located between Latitude $17^{\circ} 35^{\prime} \mathrm{N}$ and Longitude $77^{\circ} 32^{\prime} \mathrm{E}$, is the northern most city in Karnataka State, India. Its elevation is about $664 \mathrm{~m}$ above the mean sea level. The climate of Bidar and its environs is reported to be pleasant and is relatively cooler when compared to the temperatures in the neighboring districts of Karnataka. The average annual rainfall as recorded in the previous years is $916.508 \mathrm{~mm}, 919.630 \mathrm{~mm}$; the monsoon period is over four months i.e. between June to September. The highest rainfall recorded during the above period is $925.05 \mathrm{~mm}$ in July month. The average maximum temperature of $43^{\circ} \mathrm{C}$ is recorded during the period 1963 to 1979 is in the month of May. The maximum temperature however varies from $39^{\circ} \mathrm{C}$ to $43^{\circ} \mathrm{C}$ during the months of February to June and falls to $18^{\circ} \mathrm{C}$ during November and December month. The mean annual maximum humidity observed in the morning was $62.72 \%$ and the minimum observed in the evening was $43.7 \%$. Humidity will be least during the month of May. During summer the predominant wind direction is westerly, during the monsoon seasons wind blows in Southwesterly directions and during the winter seasons, the predominant wind direction is Southeasterly. Bidar urban plateau is of irregular shape, land stretching about $35.4 \mathrm{Km}$ in length and $19.3 \mathrm{Km}$ in width. The plateau consists of red laterite rocky crust, of a depth varying from $30.5 \mathrm{~m}$ to $152.4 \mathrm{~m}$ supported on impervious trap base. This has resulted in springs at the cleavages between trap and laterite rocks. Such water springs can be observed in Bidar at Gurunanak Zheera, Narasimha Zheera, Papanasha Shiva 
Temple and a few other places. The lands in Bidar and its environs drains into Manjra River (which flows in the region) is a tributary of Godavari River. The major soil types are, Red laterite soil, Black cotton soil and a combination of the above two types. The commonly used building materials (laterite stones) can be easily cut from laterite soil, once they are cut and exposed to the atmosphere, it gets harder. Ground water levels during pre-monsoon are between $2.81 \mathrm{~m}$ to $17.9 \mathrm{~m} \mathrm{bgl}$ and during post-monsoon between $0.4 \mathrm{~m}$ to $13.09 \mathrm{~m} \mathrm{bgl}$ (Groundwater information booklet 2008). Bidar urban map and its location map are presented in Figure 1 and Figure 2. The study is aimed to identify the natural and anthropogenic factors controlling the variation of ground water quality using factor analysis \& cluster analysis.

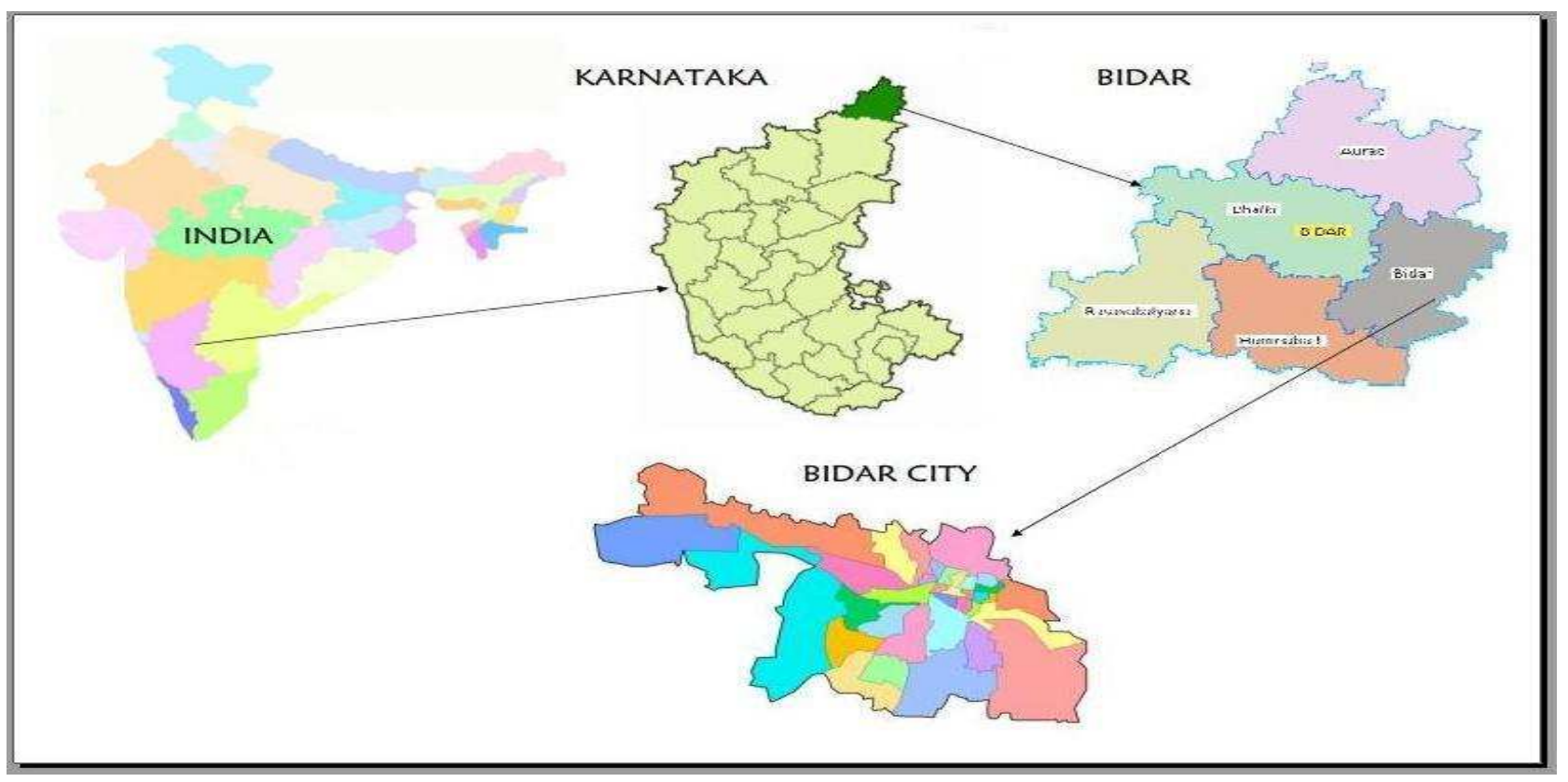

Fig1: Showing the Location of Bidar City

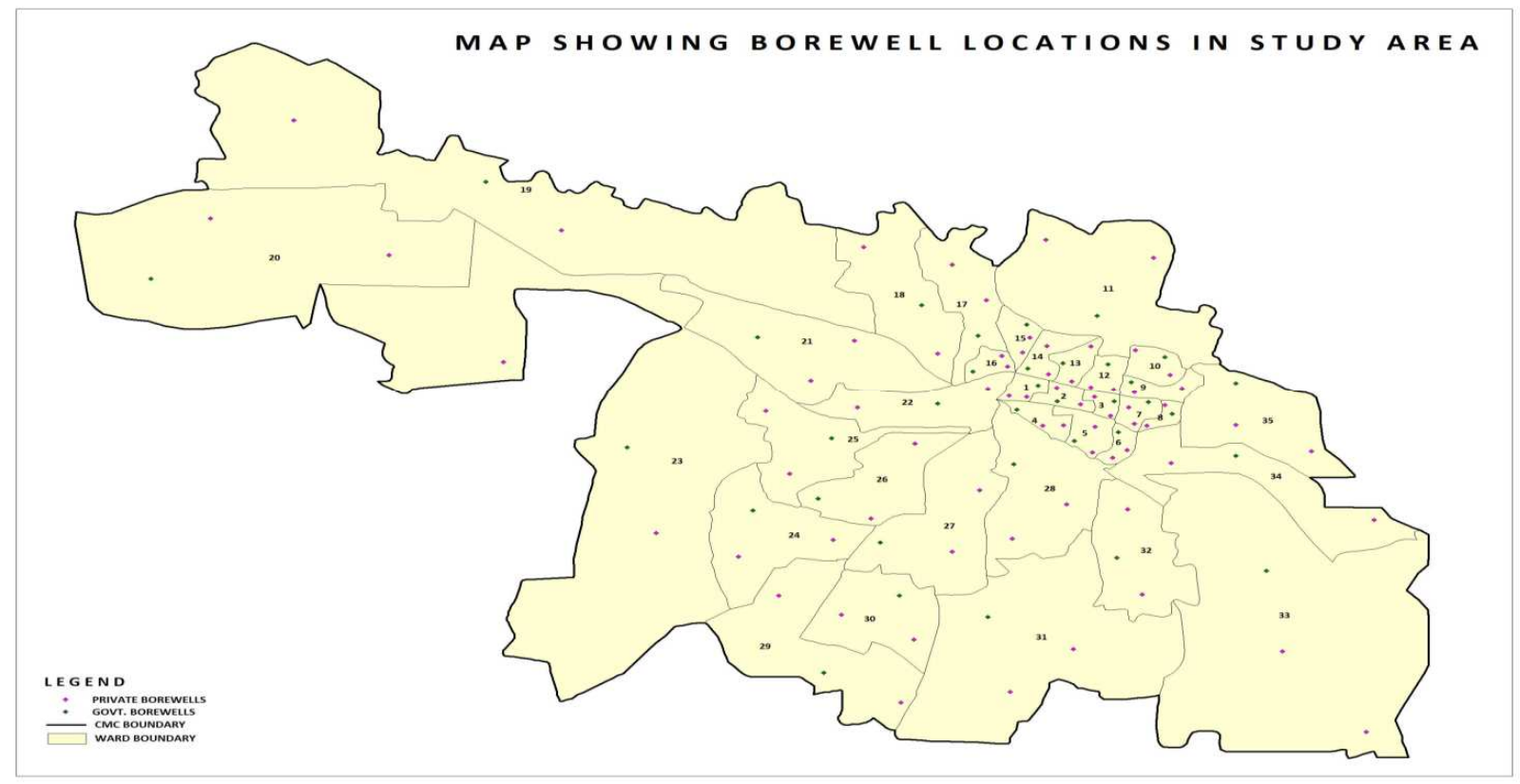

Fig2: Bidar City map showing wards and sampling locations 


\section{MATERIALS AND METHODS}

\subsection{Water Sampling and Analytical Procedures}

From each ward 3 samples, one government bore well and two residential owners bore wells water collected and analyzed so as to cover entire ward, afterwards the results average value taken to represent the particular ward. Such 35 wards analyzed covering each season (i.e., pre-monsoon \& post-monsoon) for the 3 years during 2009, $2010 \& 2012$ Total Solids (TS), Total dissolved Solids (TDS) analyzed by gravimetric method, $\mathrm{pH}$ by $\mathrm{pH}$ meter (Elico Hyderabad Make). Total hardness (TH) and $\mathrm{Ca} 2+$ as $\mathrm{CaCO} 3$ by using standard EDTA, $\mathrm{Mg} 2+$ was calculated by taking the differential value between $\mathrm{TH}$ and $\mathrm{Ca} 2+$ concentrations. $\mathrm{Na}+$ and $\mathrm{K}+$ was measured using flame photometer. Total alkalinity as $\mathrm{CaCO} 3$ which is totally $\mathrm{HCO} 3-$ as CO32- is totally absent in the entire Bidar water samples, was estimated by titrating with $\mathrm{H} 2 \mathrm{SO} 4$. Cl- was determined by titrating with standard AgNO3, SO42-, NO3-, F-, Fe, Manganese $(\mathrm{Mn})$, and Zinc ( $\mathrm{Zn})$ were analyzed by Spectrophotometer (Elico Hyderabad Make). Dissolved oxygen (DO) analyzed by Winkler's with azide modification method for statistical analysis MINITAB software is utilized.

\subsection{Factor Analysis}

Factor analysis is a very powerful technique which provides information on the meaningful parameters which describe the whole data set rendering data reduction with minimum loss of information [6]. Factor Analysis is a quantification of the significance of variables that explain the observed grouping and patterns of the inherit properties of the individual objects [7]. Factor Analysis allows the explaining of related parameters by only one factor [6, 7, and 8]. FA exposes the important factor responsible for variation in ground water quality and eventually leads to sources identification of ground water pollution. In this study, FA was applied to extract the most significant factors and to reduce the contribution of less significant variables to simplify even more of the data structure coming from factor analysis. The factors obtained were further subjected to varimax rotation according to well established rules to maximize differences between the variables and facilitate easy interpretation of the data [3, 4, and 6]. The rotating axis defined by factor analysis generates varimax factor (Factor1, Factor2....) which can further reduce the contribution of variable with minor significance.

\subsection{Cluster Analysis}

Cluster analysis is a major technique for classifying a mountain of information into manageable meaningful piles. It is a data reduction tool that creates subgroups that are more manageable than individual datum. In cluster analysis there is no prior knowledge about which elements belong to which clusters. The grouping or clusters are defined through an analysis of the data. Hierarchical CA, the most common approach, starts with each case in a separate cluster and joins the clusters together step by step until only one cluster remains $[19,20]$. The Euclidean distance usually gives the similarity between two samples, and a distance can be represented by the difference between transformed values of the samples [21].

There are four basic cluster analysis steps:

1. Data collection and selection of the variables for analysis

2. Generation of a similarity matrix

3. Decision about number of clusters and interpretation

4. Validation of cluster solution

\section{RESULTS AND DISCUSSIONS}

Summary of physico-chemical analysis of ground water of the study area for the combined pre-monsoon and post-monsoon 2009, 2010 \& 2011values are presented in the Tables $1 \& 2$. Also correlation coefficients for combined pre-monsoon and post-monsoon are worked out and presented in Tables $3 \& 4$. From the correlation table of pre-monsoon season, it is evident that a positive correlation (> 0.67) existed between $\mathrm{Ca}-\mathrm{TH}$, $\mathrm{Mg}-\mathrm{TH}, \mathrm{Na}-\mathrm{pH}, \& \mathrm{TS}-\mathrm{SO} 4$ such waters have permanent hardness and solubility of minerals. During post-monsoon season, positive correlation existed between $\mathrm{Mg}-\mathrm{TH}, \mathrm{Na}-\mathrm{TH} \&$ TS-SO4 which indicates only permanent hardness.

Table 1: Summary of combined season-wise physico- chemical composition of groundwater samples collected from the study area (covering all the 35 wards)

\begin{tabular}{|l|l|l|l|l|l|}
\hline \multirow{2}{*}{$\begin{array}{l}\text { Chemical } \\
\text { constituents }\end{array}$} & \multicolumn{6}{|l|}{ Combined Pre-monsoon of 2009, 2010 \& 2011 } \\
\cline { 2 - 6 } & Min & Max & Mean & $\sigma$ & CV \\
\hline $\mathrm{pH}$ & 7.31 & 7.94 & 7.61 & 0.156 & 2.05 \\
\hline $\mathrm{TH}$ & 101 & 120 & 108.77 & 4.685 & 4.27 \\
\hline $\mathrm{Ca} 2+$ & 80.9 & 92.1 & 85.91 & 2.643 & 3.076 \\
\hline $\mathrm{Mg} 2+$ & 18.35 & 28.25 & 22.87 & 2.63 & 11.5 \\
\hline $\mathrm{Cl}-$ & 52.85 & 176.2 & 154.73 & 20.848 & 13.47 \\
\hline $\mathrm{TDS}$ & 230.5 & 504.5 & 427.43 & 47.77 & 11.17 \\
\hline $\mathrm{Fe}+$ & 0.152 & 0.415 & 0.30 & 0.57 & 19.0 \\
\hline $\mathrm{F}-$ & 0.29 & 0.52 & 0.38 & 0.049 & 12.89 \\
\hline $\mathrm{NO} 3-$ & 16.3 & 24.6 & 20.91 & 1.688 & 8.07 \\
\hline SO42- & 21.7 & 34.5 & 27.33 & 4.456 & 18.3 \\
\hline
\end{tabular}




\begin{tabular}{|l|l|l|l|l|l|}
\hline $\mathrm{Na}+$ & 95.5 & 114.1 & 102.22 & 5.647 & 5.51 \\
\hline $\mathrm{K}+$ & 20.75 & 34.1 & 23.22 & 2.854 & 12.29 \\
\hline $\mathrm{HCO} 3-$ & 63.95 & 91.7 & 84.62 & 7.96 & 9.406 \\
\hline $\mathrm{Mn}$ & 0.026 & 0.148 & 0.09 & 0.026 & 2.88 \\
\hline $\mathrm{Zn}$ & 2.14 & 3.15 & 2.42 & 0.211 & 8.72 \\
\hline $\mathrm{DO}$ & 5.4 & 5.9 & 5.71 & 0.138 & 2.42 \\
\hline $\mathrm{TS}$ & 440.5 & 618.8 & 532.42 & 56.835 & 10.67 \\
\hline
\end{tabular}

Table 2: Summary of combined season-wise physico- chemical composition of groundwater samples collected from the study area (covering all the 35 wards)

\begin{tabular}{|l|l|l|l|l|l|}
\hline \multirow{2}{*}{$\begin{array}{l}\text { Chemical } \\
\text { constituents }\end{array}$} & \multicolumn{6}{|l|}{ Combined Post-monsoon of 2009, 2010 \& 2011 } \\
\cline { 2 - 6 } $\mathrm{pH}$ & Min & Max & Mean & $\sigma$ & $\mathrm{CV}$ \\
\hline $\mathrm{TH}$ & 1144 & 7.84 & 7.71 & 0.117 & 15.17 \\
\hline $\mathrm{Ca} 2+$ & 85.15 & 96.2 & 91.7 & 2.013 & 2.19 \\
\hline $\mathrm{Mg} 2+$ & 21.5 & 36.6 & 29.29 & 4.288 & 14.64 \\
\hline $\mathrm{Cl}-$ & 72.0 & 184.0 & 164.08 & 19.36 & 11.79 \\
\hline TDS & 411.1 & 528 & 457.93 & 29.751 & 6.49 \\
\hline Fe+ & 0.2 & 0.48 & 0.35 & 0.057 & 16.28 \\
\hline F- & 0.33 & 0.56 & 0.42 & 0.051 & 12.14 \\
\hline NO3- & 17.6 & 28 & 23.28 & 2.076 & 8.92 \\
\hline SO42- & 24.5 & 36.1 & 29.38 & 4.072 & 13.80 \\
\hline Na+ & 98.8 & 121.8 & 110.68 & 5.143 & 4.64 \\
\hline K+ & 22.85 & 30.7 & 25.34 & 2.828 & 11.16 \\
\hline HCO3- & 66 & 98.3 & 88.49 & 8.167 & 9.22 \\
\hline Mn & 0.041 & 0.193 & 0.11 & 0.034 & 30.9 \\
\hline Zn & 2.29 & 3.33 & 2.71 & 0.310 & 11.44 \\
\hline DO & 5.3 & 5.8 & 5.73 & 0.12 & 2.09 \\
\hline TS & 490 & 648.5 & 568.14 & 46.727 & 8.21 \\
\hline
\end{tabular}

\subsection{Water Pollution Sources Identification}

Factor Analysis was applied on the data set to identify the spatial sources of pollution in groundwater. According to Eigen value criterion, only factors with Eigen value greater than one are considered essential and important. Additional factors provide marginally less explanatory capability and were not examined further. In statistics, Kaiser-Meyer-Oilskin's (KMO) measure of sampling adequacy: This is an index used to examine the appropriateness of Factor Analysis. High values of F1, F2 .. (0.5 to 1.0) indicates FA is appropriate. Below $(<0.5)$ implies Factor Analysis as inappropriate. According to the Kaiser criterion, only the first three factor groups could be used, because subsequent Eigen values are less than 1. Three factors were obtained with Eigen value greater than one with total variance of $62.8 \% \& 61.6 \%$; these are considered responsible for the variation in the ground water system. Scree plot is graph of Eigen values against $\mathrm{Y}$-axis verses Factor numbers against $\mathrm{X}$-axis. Here Factor numbers with Eigen values less than 1 value are discarded and above 1 value are only considered. Scree plots for factor analysis are given in Fig No. 3 \& Fig No. 4 (Data of combined Pre-monsoon \& Postmonsoon seasons of years 2009, 2010 \& 2011). Table $5 \&$ Table 6 (Data of combined Pre-monsoon \& Post-monsoon seasons of years 2009, 2010 \& 2011) presents the factor analysis of the 17 physico-chemical parameters which constituted three factors explaining about $62.8 \%$ \& $61.6 \%$ of the total variance respectively. The classification of the factor loading as 'strong', 'moderate' and 'week' corresponds to absolute loading of $>0.75,0.75-0.50$ and $0.50-0.30$, respectively [22]. 
Table 3: Correlation coefficients for the season wise combined physico-chemical composition of groundwater samples collected from the study area (covering all the 35 Wards) for the Pre-Monsoon periods of years 2009, $2010 \& 2011$

\begin{tabular}{|c|c|c|c|c|c|c|c|c|c|c|c|c|c|c|c|c|c|}
\hline & $\mathrm{pH}$ & $\mathrm{TH}$ & $\mathrm{Ca}$ & $\mathrm{Mg}$ & $\mathrm{Cl}$ & TDS & $\mathrm{Fe}$ & $\mathrm{F}$ & NO3 & SO4 & $\mathrm{Na}$ & $\mathrm{K}$ & Alk & $\mathrm{Mn}$ & $\mathrm{Zn}$ & DO & $\begin{array}{l}\mathrm{T} \\
\mathrm{S}\end{array}$ \\
\hline $\mathrm{pH}$ & -- & -- & -- & -- & -- & -- & -- & -- & -- & -- & -- & -- & -- & -- & -- & -- & -- \\
\hline $\mathrm{TH}$ & 0.533 & -- & -- & -- & -- & -- & -- & -- & -- & -- & -- & -- & -- & -- & -- & -- & -- \\
\hline $\mathrm{Ca}$ & 0.469 & 0.857 & -- & -- & -- & -- & -- & -- & -- & -- & -- & -- & -- & -- & -- & -- & -- \\
\hline $\mathrm{Mg}$ & 0.470 & 0.905 & 0.562 & -- & -- & -- & -- & -- & -- & -- & -- & -- & -- & -- & -- & -- & -- \\
\hline $\mathrm{Cl}$ & 0.065 & 0.217 & 0.125 & 0.262 & -- & -- & -- & -- & -- & -- & -- & -- & -- & -- & -- & -- & -- \\
\hline TDS & $\begin{array}{l}- \\
0.275\end{array}$ & $\begin{array}{l}- \\
0.052\end{array}$ & 0.036 & $\begin{array}{l}- \\
0.093 \\
\end{array}$ & 0.020 & -- & -- & -- & -- & -- & -- & -- & -- & -- & -- & -- & -- \\
\hline $\mathrm{Fe}$ & $\begin{array}{l}- \\
0.417\end{array}$ & $\begin{array}{l}- \\
0.103 \\
\end{array}$ & $\begin{array}{l}- \\
0.243 \\
\end{array}$ & 0.030 & 0.297 & $\begin{array}{l}- \\
0.252 \\
\end{array}$ & -- & -- & -- & -- & -- & -- & -- & -- & -- & -- & -- \\
\hline $\mathrm{F}$ & 0.260 & 0.357 & 0.174 & 0.428 & 0.515 & $-\overline{3} 21$ & 0.458 & -- & -- & -- & -- & -- & -- & -- & -- & -- & -- \\
\hline NO3 & 0.666 & 0.444 & 0.297 & 0.480 & 0.380 & $\overline{-}-322$ & 0.061 & 0.510 & -- & -- & -- & -- & -- & -- & -- & -- & -- \\
\hline $\mathrm{SO} 4$ & 0.549 & 0.311 & 0.273 & 0.286 & 0.560 & $\begin{array}{l}- \\
0.147\end{array}$ & $\begin{array}{l} \\
0.312\end{array}$ & 0.401 & 0.487 & -- & -- & -- & -- & -- & -- & -- & -- \\
\hline $\mathrm{Na}$ & 0.672 & 0.620 & 0.441 & 0.631 & 0.461 & $-\overline{0.260}$ & $-\overline{0}-023$ & 0.552 & 0.639 & 0.641 & -- & -- & -- & -- & -- & -- & -- \\
\hline $\mathrm{K}$ & 0.454 & 0.207 & 0.226 & 0.141 & 0.418 & $\begin{array}{l}- \\
0.009\end{array}$ & $\begin{array}{l}- \\
0.205\end{array}$ & 0.276 & 0.351 & 0.521 & 0.629 & -- & -- & -- & -- & -- & -- \\
\hline Alk & 0.324 & 0.053 & 0.030 & 0.054 & 0.312 & $\begin{array}{l} \\
0.345\end{array}$ & 0.035 & 0.523 & 0.527 & 0.324 & 0.182 & 0.256 & -- & -- & -- & -- & -- \\
\hline $\mathrm{Mn}$ & $\begin{array}{l}- \\
0.176\end{array}$ & 0.096 & $-\overline{0}-087$ & $-\overline{0} 090$ & $\begin{array}{l}- \\
0.121\end{array}$ & 0.208 & $-\overline{0}-126$ & $-\overline{0}-008$ & $-\overline{0}-092$ & 0.037 & $-\overline{0.146}$ & $\begin{array}{l}- \\
0.124\end{array}$ & 0.254 & -- & -- & -- & -- \\
\hline $\mathrm{Zn}$ & $\begin{array}{l} \\
0.006\end{array}$ & $-\overline{0} 235$ & $-\overline{0} 036$ & $-\overline{0.343}$ & 0.148 & 0.329 & $-\overline{0.318}$ & $-\overline{0} 280$ & $-\overline{0} 214$ & 0.062 & $\begin{array}{l}- \\
0.086\end{array}$ & 0.289 & $\begin{array}{l}- \\
0.053\end{array}$ & 0.241 & -- & -- & -- \\
\hline DO & $\begin{array}{l}- \\
0.277\end{array}$ & $\begin{array}{l}- \\
0.182\end{array}$ & $\begin{array}{l}- \\
0.170\end{array}$ & $\begin{array}{l}-155 \\
0.15\end{array}$ & $\begin{array}{l}- \\
0.324\end{array}$ & $\begin{array}{l}- \\
0.122\end{array}$ & 0.204 & $\begin{array}{l}- \\
0.442\end{array}$ & $\begin{array}{l}- \\
0.229\end{array}$ & $\begin{array}{l}-587 \\
0.587\end{array}$ & $\begin{array}{l}-456 \\
\end{array}$ & $\begin{array}{l}- \\
0.649\end{array}$ & $\begin{array}{l}- \\
0.151\end{array}$ & $\begin{array}{l}- \\
0.118\end{array}$ & $\begin{array}{l}- \\
0.153 \\
\end{array}$ & -- & -- \\
\hline TS & 0.399 & 0.375 & 0.429 & 0.252 & 0.538 & 0.259 & $-\overline{0.156}$ & 0.367 & 0.332 & 0.741 & 0.599 & 0.641 & 0.098 & 0.063 & 0.178 & $\begin{array}{l}- \\
0.62 \\
7\end{array}$ & -- \\
\hline
\end{tabular}

Table 4: Correlation coefficients for the season wise combined physico-chemical composition of groundwater samples collected from the study area (covering all the 35 Wards) for the Post-Monsoon periods of years 2009, 2010 \& 2011

\begin{tabular}{|c|c|c|c|c|c|c|c|c|c|c|c|c|c|c|c|c|c|}
\hline & $\mathrm{pH}$ & $\mathrm{TH}$ & $\mathrm{Ca}$ & $\mathrm{Mg}$ & $\mathrm{Cl}$ & TDS & $\mathrm{Fe}$ & $\mathrm{F}$ & NO3 & SO4 & $\mathrm{Na}$ & $\mathrm{K}$ & Alk & $\mathrm{Mn}$ & $\mathrm{Zn}$ & DO & $\mathrm{TS}$ \\
\hline $\mathrm{pH}$ & -- & -- & -- & -- & -- & -- & -- & -- & -- & -- & -- & -- & -- & -- & -- & -- & -- \\
\hline TH & 0.468 & -- & -- & -- & -- & -- & -- & -- & -- & -- & -- & -- & -- & -- & -- & -- & -- \\
\hline $\mathrm{Ca}$ & 0.463 & 0.610 & -- & -- & -- & -- & -- & -- & -- & -- & -- & -- & -- & -- & -- & -- & -- \\
\hline $\mathrm{Mg}$ & 0.353 & 0.938 & 0.297 & -- & -- & -- & -- & -- & -- & -- & -- & -- & -- & -- & -- & -- & -- \\
\hline $\mathrm{Cl}$ & 0.154 & 0.072 & 0.131 & 0.040 & -- & -- & -- & -- & -- & -- & -- & -- & -- & -- & -- & -- & -- \\
\hline TDS & $\begin{array}{l}- \\
0.152\end{array}$ & $\begin{array}{l}- \\
0.186\end{array}$ & 0.001 & $\begin{array}{l}- \\
0.222 \\
\end{array}$ & 0.011 & -- & -- & -- & -- & -- & -- & -- & -- & -- & -- & -- & -- \\
\hline $\mathrm{Fe}$ & $-\overline{0.413}$ & $-\overline{0}-052$ & $-\overline{0}-337$ & 0.093 & 0.340 & $\begin{array}{l}- \\
0.123 \\
\end{array}$ & -- & -- & -- & -- & -- & -- & -- & -- & -- & -- & -- \\
\hline $\mathrm{F}$ & 0.207 & 0.294 & 0.058 & 0.330 & 0.479 & $\begin{array}{l}- \\
0.341 \\
\end{array}$ & 0.509 & -- & -- & -- & -- & -- & -- & -- & -- & -- & -- \\
\hline NO3 & 0.600 & 0.531 & 0.436 & 0.446 & 0.323 & $\begin{array}{l}- \\
0.347 \\
\end{array}$ & $\begin{array}{l} \\
0.037\end{array}$ & 0.449 & -- & -- & -- & -- & -- & -- & -- & -- & -- \\
\hline SO4 & 0.529 & 0.181 & 0.388 & 0.048 & 0.505 & $\begin{array}{l}- \\
0.232 \\
\end{array}$ & $\begin{array}{l}- \\
0.279 \\
\end{array}$ & 0.349 & 0.393 & -- & -- & -- & -- & -- & -- & -- & -- \\
\hline $\mathrm{Na}$ & $\begin{array}{l}- \\
0.631\end{array}$ & 0.701 & 0.423 & 0.657 & 0.228 & $\begin{array}{l}- \\
0.431\end{array}$ & 0.057 & 0.506 & 0.723 & 0.401 & -- & -- & -- & -- & -- & -- & -- \\
\hline $\mathrm{K}$ & 0.427 & 0.120 & 0.324 & 0.005 & 0.423 & $\begin{array}{l}- \\
0.020\end{array}$ & $\begin{array}{l}- \\
0.166\end{array}$ & 0.221 & 0.192 & 0.462 & 0.335 & -- & -- & -- & -- & -- & -- \\
\hline Alk & 0.450 & 0.071 & 0.054 & 0.056 & 0.415 & - & 0.061 & 0.479 & 0.567 & 0.403 & 0.373 & 0.277 & -- & -- & -- & -- & -- \\
\hline
\end{tabular}




\begin{tabular}{|c|c|c|c|c|c|c|c|c|c|c|c|c|c|c|c|c|c|}
\hline & & & & & & 0.280 & & & & & & & & & & & \\
\hline $\mathrm{Mn}$ & 0.118 & $\overline{-}-\overline{0} 12$ & $\overline{0}-136$ & 0.041 & $-\overline{0}$ & 0.183 & $-\bar{l}-172$ & 0.123 & 0.046 & 0.121 & 0.005 & $-\overline{0}-036$ & 0.265 & -- & -- & -- & -- \\
\hline $\mathrm{Zn}$ & 0.050 & $\begin{array}{l}- \\
0.465 \\
\end{array}$ & $\begin{array}{l} \\
0.119\end{array}$ & $\begin{array}{l}- \\
0.506 \\
\end{array}$ & 0.142 & 0.367 & $\begin{array}{l}- \\
0.300 \\
\end{array}$ & $\begin{array}{l}- \\
0.248 \\
\end{array}$ & $\begin{array}{l}- \\
0.291 \\
\end{array}$ & $\begin{array}{l} \\
0.011 \\
\end{array}$ & $\begin{array}{l}- \\
0.337\end{array}$ & 0.383 & 0.014 & 0.326 & -- & -- & -- \\
\hline DO & $\begin{array}{l}- \\
0.300\end{array}$ & $\overline{-} \mathbf{0 . 1 0 2}$ & $\overline{-} \mathbf{0 . 0 7 6}$ & $\overline{-} \mathbf{0 . 0 8 8}$ & $\overline{0}-200$ & 0.065 & 0.160 & $\overline{-} \mathbf{0 . 3 7 0}$ & $\overline{-} \mathbf{0 . 1 3 6}$ & $\overline{-} \mathbf{0 . 4 1 2}$ & $\overline{0}-222$ & $\overline{0}-532$ & $\overline{-} \mathbf{0 . 1 9 9}$ & $\begin{array}{l}- \\
0.194\end{array}$ & $\begin{array}{l}- \\
0.07 \\
5\end{array}$ & -- & -- \\
\hline TS & 0.491 & 0.199 & 0.439 & 0.050 & 0.538 & 0.033 & 0.007 & 0.411 & 0.222 & 0.727 & 0.367 & 0.629 & 0.242 & 0.106 & $\begin{array}{l}0.16 \\
1\end{array}$ & $\begin{array}{l}- \\
0.43 \\
5\end{array}$ & -- \\
\hline
\end{tabular}

Scree Plot of $\mathrm{C} 1-\mathrm{C} 17$

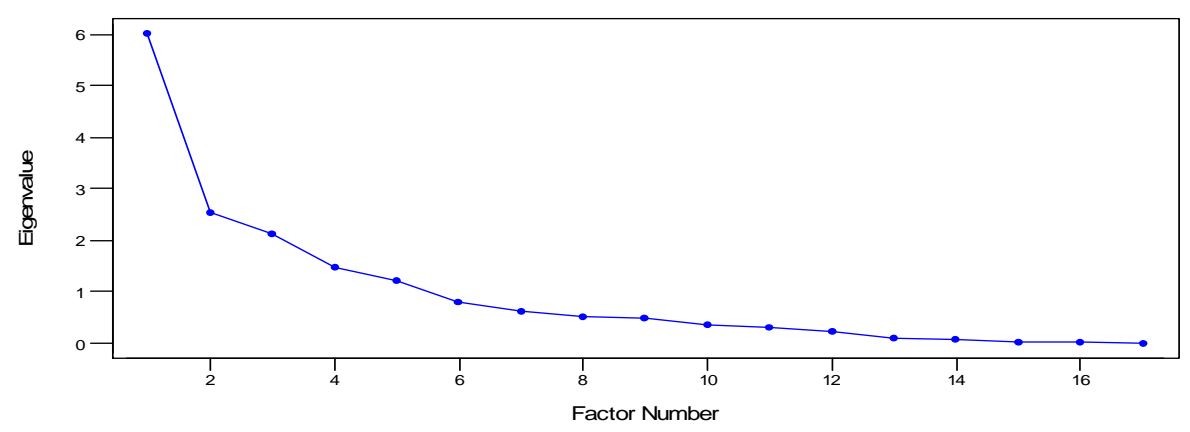

Fig3: Screen Plot for the combined data of pre-monsoon 2009, 2010 \& 2011

Table 5: Factor Analysis (for Combined Data of Pre-Monsoon 2009, 2010 \& 2011) (Rotated Factor Loadings and Communalities Varimax Rotation)

\begin{tabular}{|l|l|l|l|l|}
\hline Variable & Factor 1 & Factor 2 & Factor 3 & Communality \\
\hline $\mathrm{pH}$ & 0.429 & 0.656 & -0.001 & 0.694 \\
\hline $\mathrm{TH}$ & 0.161 & 0.930 & 0.031 & 0.892 \\
\hline $\mathrm{Ca} 2+$ & 0.168 & 0.799 & -0.198 & 0.706 \\
\hline $\mathrm{Mg} 2+$ & 0.125 & 0.842 & 0.206 & 0.767 \\
\hline $\mathrm{Cl}-$ & 0.666 & 0.001 & 0.317 & 0.543 \\
\hline $\mathrm{TDS}$ & 0.044 & -0.145 & -0.661 & 0.459 \\
\hline $\mathrm{Fe}+$ & -0.157 & -0.218 & 0.737 & 0.616 \\
\hline $\mathrm{F}-$ & 0.511 & 0.198 & 0.683 & 0.767 \\
\hline $\mathrm{NO3-}$ & 0.463 & 0.468 & 0.457 & 0.643 \\
\hline $\mathrm{SO} 42-$ & 0.806 & 0.259 & 0.019 & 0.718 \\
\hline $\mathrm{Na}+$ & 0.623 & 0.600 & 0.214 & 0.794 \\
\hline $\mathrm{K}+$ & 0.793 & 0.131 & -0.110 & 0.659 \\
\hline $\mathrm{HCO3-}$ & 0.446 & -0.057 & 0.494 & 0.446 \\
\hline Mn & 0.149 & -0.270 & -0.177 & 0.127 \\
\hline Zn & 0.345 & -0.353 & -0.563 & 0.561 \\
\hline DO & -0.771 & -0.050 & 0.127 & 0.613 \\
\hline TS & 0.808 & 0.251 & -0.197 & 0.754 \\
\hline Eigen Value & 6.1 & 2.5 & 2.2 & -- \\
\hline$\%$ Variance & 26.2 & 21.6 & 15.0 & -- \\
\hline $\begin{array}{l}\text { Cumulative } \\
\text { Variance }\end{array}$ & 26.2 & 47.8 & 62.8 & -- \\
\hline & & & & \\
\hline & & & & \\
\hline
\end{tabular}




\subsection{Governing Factors Of Groundwater Quality By}

Factor Analysis (For Combined Data Of Pre-Monsoon

2009, 2010 \& 2011) (Rotated Factor Loadings And

Communalities Varimax Rotation):

\section{Factor1:}

Having Eigen value with 6.1 and $26.2 \%$ of the variance, has high loadings on $\mathrm{SO}_{4}, \mathrm{~K} \& \mathrm{TS}$, moderate loadings on $\mathrm{Cl}, \mathrm{F} \&$ $\mathrm{Na}$ and low loadings on $\mathrm{pH}, \mathrm{NO}_{3}, \mathrm{HCO}_{3} \& \mathrm{Zn}$.

(1) High loading: It suggests that the quality of ground water is mainly controlled by high loading parameters. The high $\mathrm{SO}_{4}$ is related to the long-history of evaporation process, the high value of $\mathrm{K}$ suggests pollution from application of potash fertilizers to agricultural lands (Cain et al., 1989) and high loading on TS accounts for low mixing of overlying soft soil after percolation due to monsoon runoff/pollution.

(2) Moderate loading: Gentle slope and sluggish-drainage conditions supporting longer-residence time of groundwater, more water-rock interaction and higher solubility of minerals mark mostly saline water and enrichment of $\mathrm{Na}$ and $\mathrm{Cl}$. F indicates weathering of country rocks.
(3) Low loading: The combination of $\mathrm{pH}, \mathrm{NO}_{3}, \mathrm{Zn} \& \mathrm{HCO}_{3}$ indicates weathering of country rocks.

\section{Factor 2:}

Having Eigen value with 2.5 and $21.6 \%$ of the variance, has high loadings of $\mathrm{TH}, \mathrm{Ca} \& \mathrm{Mg}$, moderate loadings on $\mathrm{pH} \& \mathrm{Na}$ and low loadings on $\mathrm{NO}_{3}$.

(1) High loading: The combination of $\mathrm{TH}, \mathrm{Ca} \& \mathrm{Mg}$ represents permanent hardness.

(2) Moderate loading: $\mathrm{Na}$ with $\mathrm{pH}$ represents solubility of minerals.

(3) Low loading: Presence of $\mathrm{NO}_{3}$ indicates mild pollution

\section{Factor 3:}

Having Eigen value with 2.2 and $15.0 \%$ of variance, has no high loadings, moderate loadings on $\mathrm{Fe} \& \mathrm{~F}$, and low loadings on $\mathrm{HCO}_{3} \& \mathrm{NO}_{3}$.

(1) High loading: No loading at all

(2) Moderate loading: Fe \& F indicates weathering of country rocks.

(3) Low loading: Presence of $\mathrm{HCO}_{3} \& \mathrm{NO}_{3}$ weathering of country rocks

\section{Scree Plot of $\mathrm{C} 1-\mathrm{C} 17$}

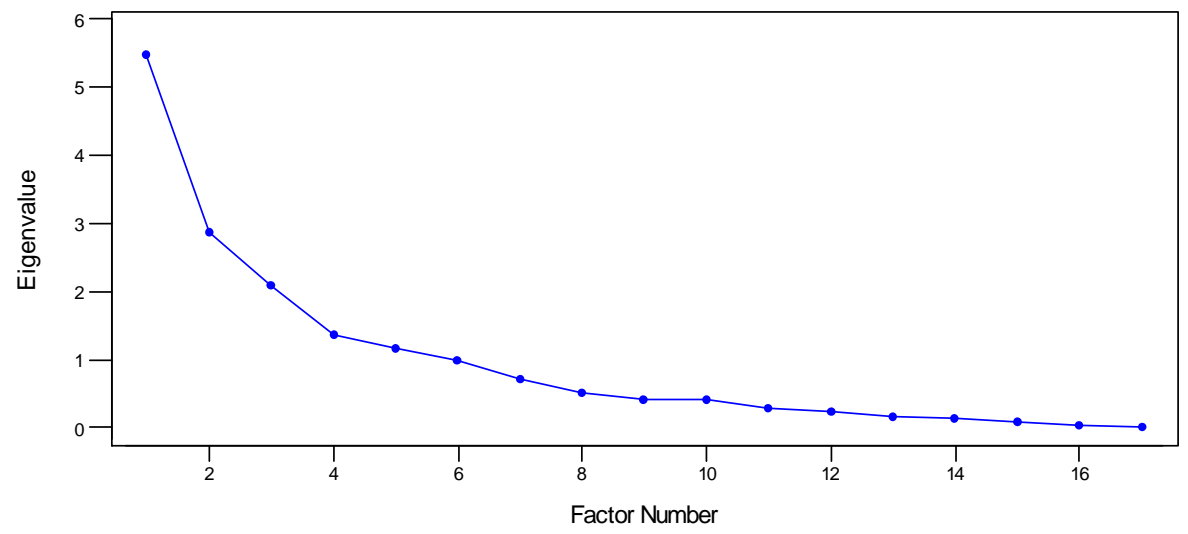

Fig4: Screen Plot for the combined data of pre-monsoon 2009, 2010 \& 2011

Table 6: Factor Analysis (for Combined Data of Post-Monsoon 2009, 2010 \& 2011) (Rotated Factor Loadings and Communalities Varimax Rotation)

\begin{tabular}{|l|l|l|l|l|}
\hline Variable & Factor 1 & Factor 2 & Factor 3 & Communality \\
\hline $\mathrm{pH}$ & 0.569 & -0.582 & 0.252 & 0.725 \\
\hline $\mathrm{TH}$ & 0.922 & -0.023 & 0.058 & 0.854 \\
\hline $\mathrm{Ca} 2+$ & 0.592 & -0.320 & 0.383 & 0.600 \\
\hline $\mathrm{Mg} 2+$ & 0.848 & 0.112 & -0.103 & 0.742 \\
\hline $\mathrm{Cl}-$ & -0.010 & -0.615 & -0.487 & 0.615 \\
\hline
\end{tabular}




\begin{tabular}{|l|l|l|l|l|}
\hline TDS & -0.405 & -0.022 & 0.398 & 0.323 \\
\hline Fe+ & -0.089 & 0.222 & -0.860 & 0.796 \\
\hline F- & 0.317 & -0.385 & -0.735 & 0.789 \\
\hline NO3- & 0.700 & -0.335 & -0.204 & 0.644 \\
\hline SO42- & 0.255 & -0.756 & -024 & 0.652 \\
\hline $\mathrm{Na}+$ & 0.814 & -0.321 & -0.203 & 0.806 \\
\hline $\mathrm{K}+$ & 0.056 & -0.781 & 0.086 & 0.620 \\
\hline Alk(HCO3-) & 0.186 & -0.525 & -0.392 & 0.464 \\
\hline $\mathrm{Mn}$ & -0.118 & -0.281 & 0.075 & 0.098 \\
\hline $\mathrm{Zn}$ & -0.604 & -0.441 & 0.349 & 0.682 \\
\hline $\mathrm{DO}$ & -0.066 & 0.596 & 0.036 & 0.360 \\
\hline TS & 0.144 & -0.813 & -0.057 & 0.684 \\
\hline Eigen Value & 5.5 & 2.9 & 2.0 & -- \\
\hline$\%$ Variance & 24.6 & 23.6 & 13.4 & -- \\
\hline $\begin{array}{l}\text { Cumulative \% of } \\
\text { Variance }\end{array}$ & 24.6 & 48.2 & 61.6 & -- \\
\hline
\end{tabular}

\subsection{Governing Factors Of Groundwater Quality By}

Factor Analysis (For Combined Data Of PostMonsoon 2009, 2010 \& 2011) (Rotated Factor

\section{Loadings And Communalities Varimax Rotation):}

\section{Factor1:}

Having Eigen value with 5.5 and $24.6 \%$ of the variance, has high loadings on $\mathrm{TH}, \mathrm{Mg} \& \mathrm{Na}$, moderate loadings on $\mathrm{Ca}, \mathrm{pH}$ $\& \mathrm{NO}_{3}$ and low loadings on $\mathrm{F}$.

(1) High loading: It suggests that the quality of ground water is mainly controlled by high loading parameters. The high loadings on $\mathrm{TH}, \mathrm{Mg} \& \mathrm{Na}$ shows the permanent hardness of groundwater

(2) Moderate loading: The combination of $\mathrm{pH}, \mathrm{NO}_{3} \& \mathrm{HCO}_{3}$ indicates weathering of country rocks.

(3) Low loading: F indicates weathering of country rocks.

\section{Factor 2:}

Having Eigen value with 2.9 and $23.6 \%$ of the variance, has no high loadings at all, moderate loadings on DO and no low loadings too

(1) High loading: No loading at all

(2) Moderate loading: DO indicate mild reaeration/mixing of rain water.

(3) Low loading: No loading at all

\section{Factor 3:}

Having Eigen value with 2.0 and $13.4 \%$ of variance, has no high loadings, no moderate loadings and low loadings on $\mathrm{Ca}$, TDS \& Zn

(1) High loading: No loading at all

(2) Moderate loading: No loading at all

(3) Low loading: Presence of $\mathrm{Ca}$ indicates weathering of country rocks, whereas TDS \& $\mathrm{Zn}$ indicates mild pollution

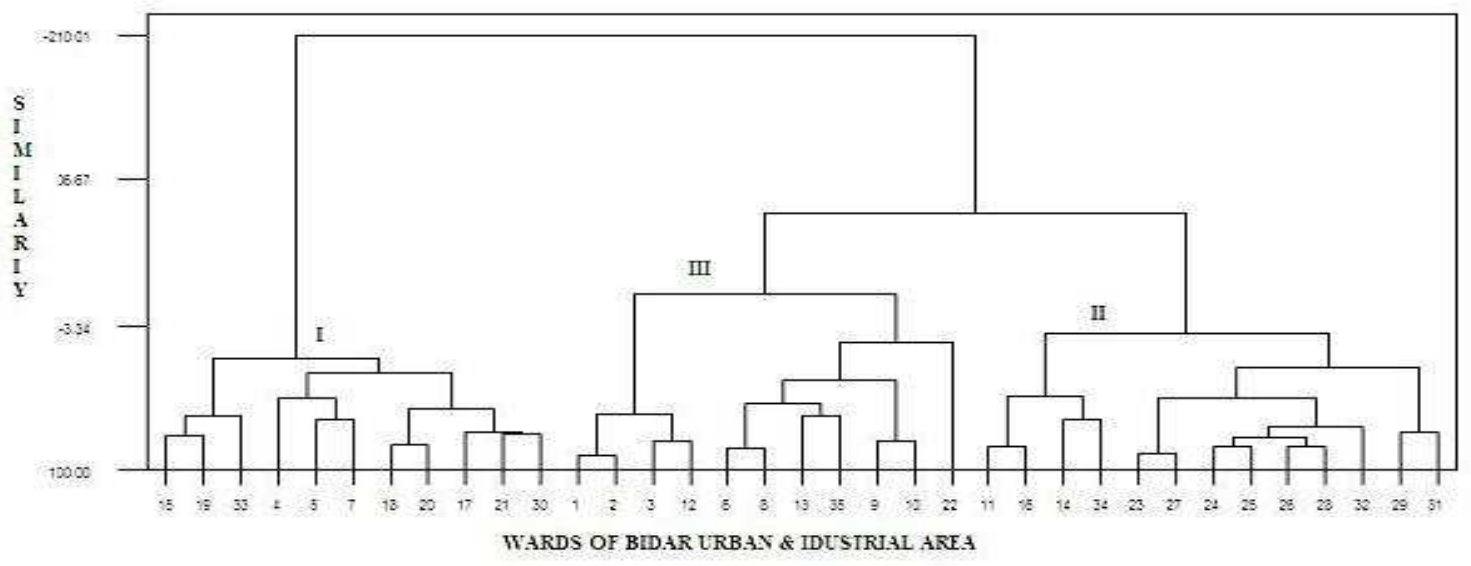

Fig5: Dendograms of hierarchical cluster analysis for combined pre-monsoon 2009, 2010 \& 2011 


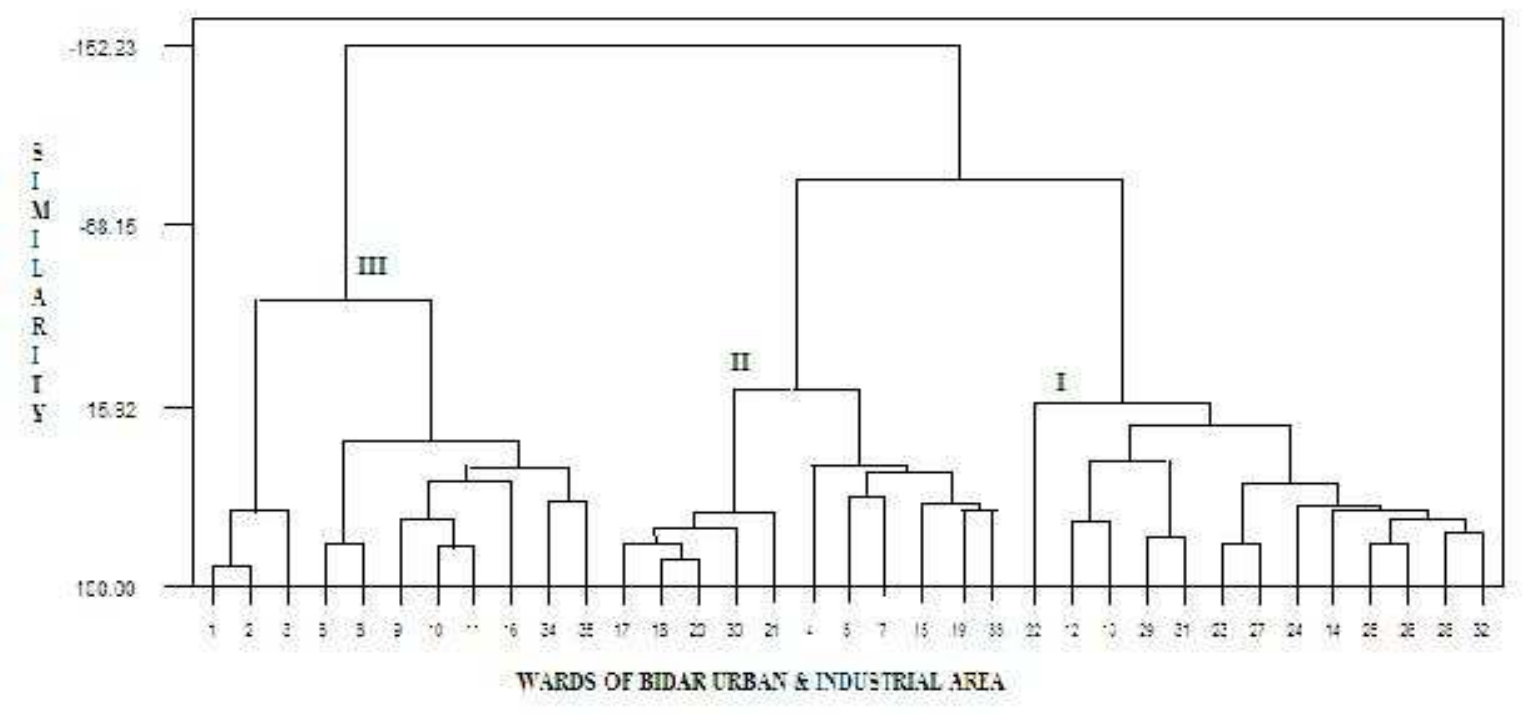

Fig6: Dendograms of hierarchical cluster analysis for combined post-monsoon 2009, 2010 \& 2011

\subsection{Cluster Analysis:}

In this study, sampling site classification as performed by the use of cluster analysis. Hierarchical Cluster Analysis was performed on the factor scores obtained from factor analysis using Ward's method with squared Euclidean distances as a measure of similarity. The linkage method that will determine how the distance between two clusters is defined. Choices include Single, Average, Centroid, Complete, McQuitty, Median, and Ward [23]. Cluster analysis from factor scores of stations reduce the clustering error caused by data error or multicollinearity. Ward's method uses analysis of variance (ANOVA) to calculate the distances between clusters to minimize the sum of squares of any two possible clusters at each step. Results of cluster analysis are represented using dendogram. In the dendograms of Bidar city water sampling stations are classified into three clusters as shown in Fig. 5 \& 6.

On the basis of Cluster analysis, wards of Bidar urban are divided as follows (Combined pre-monsoon 2009, 2010 \& 2011): Cluster-I(Ward4,5,7,15,17,18,19,20,21,30 \& 33), Cluster-II (Ward 11,14,16,23,24,25,26,27,28,29,31 \& 32), Cluster-III (Ward 1,2,3,6,8,9,10,12,13 \& 35).

And on the basis of Cluster analysis, wards of Bidar urban are divided as fallows (Combined post-monsoon 2009, 2010 \& 2011): Cluster-I (Ward $12,13,14,22,23,24,25,26,27,28,29,31,32)$, Cluster-II (Ward $4,5,7,15,17,18,19,20,21,30,33), \quad$ Cluster-III $\quad$ (Ward $1,2,3,6,8,9,10,11,1634,35)$.

Wards of same clusters have the similar pattern of the groundwater quality.
Combined pre-monsoon 2009, $2010 \&$ 2011: Wards of ClusterI are more polluted, here major contributors in ground water pollution are SO4, K \& TS. Wards of Cluster-II are less polluted areas. These wards have higher loading in $\mathrm{TH}, \mathrm{Ca}$, and $\mathrm{Mg}$ which indicates permanent hardness. Wards of Cluster-III are moderately polluted. Pollution in these wards is mainly by Fe \& F which is generally by weathering of country rocks.

Combined post-monsoon 2009, 2010 \& 2011: Wards of Cluster-I are more polluted, here $\mathrm{TH}, \mathrm{Mg} \& \mathrm{Na}$ are having higher contribution. Wards of Cluster-II which are less polluted areas also here mild reaeration is taking place. Wards of Cluster-III are moderately polluted. Pollution in these wards is mainly due to $\mathrm{Ca}, \mathrm{Zn}$ and TDS. $\mathrm{Ca} \& \mathrm{Zn}$ indicates weathering of country rocks. TDS may be by percolation of runoff and soft overlying soil during monsoon.

\section{CONCLUSIONS}

Factor analysis was successfully applied to identify the sources of pollution in Bidar urban and its industrial area. The results reveal that among the variables studied $\mathrm{TH}, \mathrm{Ca}, \mathrm{Mg}$, are statistically the most significant parameters that bring variation in water quality in the area. During pre-monsoon period of analysis, $\mathrm{TH}, \mathrm{Ca}, \& \mathrm{Mg}$ are dominants, TS \& $\mathrm{K}$ are present moderately. SO4 also present in low. Whereas during postmonsoon period of analysis, again $\mathrm{TH} \& \mathrm{Mg}$ are dominants but remaining parameters viz., K, TS, SO4 \& TDS have low loadings. This may be due to dilution of ground water during monsoon season. The contribution of anthropogenic activities through discharge of domestic waste water/industrial effluent into the groundwater is very less as is identified by Factor Analysis. To restore the ecological stability and aesthetic 
quality of ground water, there is need to empower local environmental agency to enforce regular compliance to the standard guidelines to the various industries located around the area and safe disposal of domestic waste water. Cluster analysis through factor scores and by dendograms, identified low, medium and high polluted wards during both pre-monsoon and post-monsoon periods of the study area. It has been revealed that hardness is more during pre-monsoon studies and little less during post-monsoon studies. In pre-monsoon period, SO4 is major contributor, $\mathrm{TH}, \mathrm{Ca} \& \mathrm{Mg}$ present mildly high showing permanent hardness to water whereas Fe \& F which are moderately present which is generally by weathering of country rocks. In post-monsoon period, $\mathrm{TH}, \mathrm{Mg} \& \mathrm{Na}$ are major contributors. $\mathrm{Ca}, \mathrm{Zn}$ and TDS are moderately present. Ca \& Zn indicates weathering of country rocks. TDS may be by percolation of runoff and soft overlying soil during monsoon. DO is present in few wards which indicates mild reaeration by percolated water during monsoon. In all since factors scores are less than 0.35 value pollution is less in the entire Bidar urban.

\section{ACKNOWLEDGEMENTS}

The authors sincerely thank "Hyderabad Karnataka Education Society" Gulbarga, Karnataka State, India

\section{REFERENCES}

[1] Reza, R. and G. Singh, Heavy Metal Contamination and its Indexing Approach for River Water. International Journal of Environmental Science and Technology, 7(4): 785-792, (2010).

[2] Cachada A., M.E. Pereira, E. Ferreira and A.C. Duarte, Sources of Potentially Toxic Elements and Organic Pollutants in an Urban Area Subjected to an Industrial Impact, Journal of Environmental Monitoring and Assessment, DOI: 10.1007/s 10661-011-1943-8, (2011)

[3] Singh, K.P., A. Malik, D. Mohan, S. Sinha and K.S. Vinod, Chemometric Data Analysis of Pollutants in wastewater - a Case Study. Analytica Chemica Acta., 532(1): 15-25, (2005).

[4] Shrestha, S. and F. Kazama, "Assessment of Surface Water Quality using Multivariate Statistical Techniques: A Case Study of the Fuji River Basin, Japan." Environmental Modelling \& Software, 22(4): 464-475, (2007)

[5] Qadir, A.R., N. Malik and S.Z. Husain, Spatio-Temporal Variations in Water Quality of Nullah Aik-Tributary of the River Chenab, Pakistan. Environmental Monitoring and Assessment, 140(1): 43-59, (2008).

[6] Singh, K.P., A. Malik, D. Mohan and S. Sinha, Multivariate Statistical Techniques for the Evaluation of Spatial and Temporal Variations in Water Quality of Gomati River (India) - a Case Study. Water Research, 38 (18): 3980-3992, (2004)

[7] Kowalkowski T., R. Zbytniewski, J. Szpejna and B. Buszewski, Application of Chemometrics in River
Water Classification, Journal of Water Research, 40(4): 744-752, (2006)

[8] Boyacioglu. H. and H. Boyacioglu, "Water Pollution Sources Assessment by Multivariate Statistical Methods in the Tahtali Basin, Turkey." Journal of Environmental Geology, 54(2): 275-282, (2006)

[9] UNESCO; Groundwater in Hard rock, Studies and Reports in Hydrology, 32, Paris, 228 p; 1984.

[10] Usunoff, E.J. and A.G. Guzman; Multivariate analysis in Hydrochemistry: an example of use of factor and correspondence analysis. Ground Water, Vol. 27, pp. 27-34; (1989)

[11] Subba Rao, N., Prakasa Rao, J., Chandra Rao, P., Niranjan Babu, P. and G. Krishna Rao; Hydrogeochemical zoning in crystalline terrain and its significance to water quality. J. Geol. Soc. India, Vol. 49, pp. 715-719; (1997).

[12] Subba Rao, N. and G. Krishna Rao; Intensity of pollution of groundwater in Visakhapatnam area, Andhra Pradesh J. Geol. Soc. India, Vol. 36, pp. 670673; (1990)

[13] Somasundaram, M.V., Ravindran, G. and J.H. Tellam; Groundwater pollution of the Madras urban aquifer, India. Ground Water, Vol. 31, pp. 4-11; (1993)

[14] Drever, J.I.; The Geochemistry of Natural Waters. Prentice-Hill, Inc., New York, 462 p; (1988).

[15] Karanth, K.R.; Impact of human activities on hydro geological environment. J. Geol. Soc. India, Vol. 38, pp. 195-206; (1991).

[16] Datta, P.S. and S.K. Tyagi; Major ion chemistry of groundwater in Delhi area: Chemical weathering process and groundwater flow regime. J. Geol. Soc. India, Vol. 47, pp. 179-188; (1996).

[17] Ballukraya, P.N. and R. Ravi; Characterization of groundwater in the unconfined aquifers of Chennai City, India. J. Geol. Soc. India, Vol. 54, pp. 13-22; (1999)

[18] Mohan, R., Singh, A.K., Tripathi, J.K. and G.C. Chowdhary; Hydrochemistry and quality assessment of groundwater in Naini Industrial Area, Allahabad district, Uttar Pradesh. J. Geol. Soc. India, Vol. 55, pp. 77-89; (2000).

[19] J. Lattin, D. Carroll and P. Green, Analyzing multivariate data New York: Duxbury, (2003).

[20] J. McKenna, Environmental Modeling and Software, 18, 205, (2003)

[21] M. Otto, R. Kellner, J. M. Mermet, and H. M. Widmer, (Eds.), Multivariate methods in Analytical chemistry. Weinheim: Wiley-VCH. (1998).

[22] Liu. C.W.; Lin, K.H.; Kuo, Y.M. Application of factor analysis in the assessment of groundwater quality in a Blackfoot disease area in Taiwan. The Science of Total Environment, Vol. 313, pp77-89, (2003).

[23] Lance and W.T. Williams, a General Theory of Classificatory Sorting Strategies, I. Hierarchical systems, Computer Journal, 9, 373380, (1967). 